\title{
Distributed Event-Triggered Circle Formation Control for Multiagent Systems with Nonuniform Quantization
}

\author{
Jiayan Wen $\mathbb{D}^{1},{ }^{1,2}$ Haijiang Zhang $\mathbb{D}^{1},{ }^{1,2}$ Guangxing Tan, ${ }^{1}$ Ning Cai, ${ }^{3}$ and Guangming Xie ${ }^{1,4}$ \\ ${ }^{1}$ School of Electrical and Information Engineering, Guangxi University of Science and Technology, Liuzhou 545006, China \\ ${ }^{2}$ Guangxi Key Laboratory of Automobile Components and Vehicle Technology in Guangxi University of Science and Technology, \\ Liuzhou, China \\ ${ }^{3}$ College of Artificial Intelligence, Beijing University of Posts and Telecommunications, Beijing 100876, China \\ ${ }^{4}$ College of Engineering, Peking University, Beijing 100871, China
}

Correspondence should be addressed to Jiayan Wen; wenjiayan2012@126.com

Received 10 December 2020; Accepted 28 July 2021; Published 9 August 2021

Academic Editor: Abdellatif Ben Makhlouf

Copyright (C) 2021 Jiayan Wen et al. This is an open access article distributed under the Creative Commons Attribution License, which permits unrestricted use, distribution, and reproduction in any medium, provided the original work is properly cited.

\begin{abstract}
This article focuses on circle formation control problem of multiagent systems based on event-triggered strategy under limited communication bandwidth. In such system, each agent can only perceive the angular distance of its nearest neighbor in the counterclockwise direction, and the angular distance of the nearest neighbor in the clockwise direction needs to be obtained by communicating with each other. In order to address the aforementioned problem, a novel distributed algorithm based on the combination of nonuniform quantitative communication technology and event-triggered control is proposed. Sufficient conditions on circle formation control are derived under which the states of all agents can be confirmed to converge to some desired equilibrium point. Different from the traditional uniform quantization communication framework, nonuniform quantization can be beneficial for handling small signals and improving the performance of multiagent systems concerned. Furthermore, under the proposed policy, all the designed quantizers do not emerge saturated. Numerical simulation results are provided to verify the effectiveness of the proposed algorithm.
\end{abstract}

\section{Introduction}

According to the new research literature, formation control of multiagent systems (MASs), which is oriented to design an appropriate control protocol so that all agents can maintain a prescribed geometric shape, has received significant attention owing to its broad applications [1-3]. Circle formation control as a datum problem in formation control of MASs is widely investigated in sundry areas [4-6]. In many actual application scenarios, the components of the system will be scattered in a wide area, and the exchange of information between components (including controllers, actuators, and wireless sensors) is mainly implemented via digital communication networks [7]. In theoretical analysis, the system is usually regarded as an ideal state. More specifically, for this ideal situation, it is assumed that each agent does not experience packet loss and distortion when performing information interaction with its neighbors [8]. The requirement of the basic assumption is that all communication channels between agents possess a sufficiently large bandwidth or unlimited capacity. However, it is contradictory to actual system applications, that is to say, for the practical application case, digital network resources often may be limited by different degrees of energy and communication bandwidth due to some reasons [9]. In particular, when it comes up to the large-scale MASs, the limitation of network communication bandwidth will not only affect the quality of data transmission but also cause the overall performance of the system to decrease. As a result, considering the limited communication capacity of the whole digital network, it is necessary to pay much attention when designing the control protocols with close to practical applications.

In view of the limitation of network resources of MASs, a variety of control algorithms involving information quantification have gradually emerged. In the pioneering works 
$[10,11]$, the quantization communication based on integervalued and real-valued has been analyzed, respectively. Aiming at the shortcomings of the static quantizer designed in $[10,11]$, the authors of [12] have further proposed a dynamic encoder-decoder quantization algorithm. Then, $\mathrm{Li}$ et al. [13] characterized a novel control method that can be symmetrically compensated by adjusting the corresponding parameters of the controller to reduce the number of bits transmitted by each digital channel to only 1 bit. Thereafter, the theory that is closely related to the quantization algorithm of networked systems has been systematically studied in [14], where the authors provided a comprehensive analysis of the average consensus of MASs with a finite number of quantization levels by introducing a scaling function into the encoding-decoding quantizer. The extensions of the authors' work in [13] are further investigated for cases: the agents being governed by the second-order linear dynamics $[15,16]$, discrete linear systems $[17,18]$, and nonlinear systems $[19,20]$, respectively, the presence of communication channel time-delays, and so on. $\mathrm{Xu}$ et al. [21] have considered the leader-following fixed-time quantitative consensus problem of the nonlinear multiagent systems with a novel way of impulse control.

Owing to the limited bandwidth and energy resource, circle formation control of multiagent systems over directed graph with quantized communications is significant from both theoretical and engineering points of view. Furthermore, as the questions arising pointed out in [14], the designed protocols in the existing literature aforementioned are executed synchronously, and they need to be updated at each time step. This may cause much unnecessary energy consumption, especially in an environment with limited resources. With the growing demand in industry on systematic methods to model, analyze, and design systems, event-triggered control (ETC) has been proposed as a promising control mode to solve the above problems $[22,23]$. Fortunately, this ETC framework has been applied to deal with the quantized consensus problems of MASs. In $[24,25]$, the authors introduced ETC into the second-order system, which proved that it can effectively reduce the calculation amount of the system and reduce the update frequency of the controller. In [26], a nonlinear decomposition method of asymmetric hysteresis quantizer is proposed by using the fuzzy logic system to estimate random perturbation term and unknown nonlinear function, and the sector constraint property is used. In [27-30], the authors have investigated the quantized consensus problem of the general linear systems and nonlinear systems, respectively, with the couple of ETC fashion. To the best knowledge of the authors, most studies have focused on the analysis and design of uniform quantification combined with ETC strategy to solve the formation control problem of MASs, and few results are devoted to concentrate on the co-design structure between nonuniform quantification and ETC scheme compared with the former counterpart, especially few for the datum problem of circle formation. Thus, it is natural to motivate us to consider the novel combination involving nonuniform quantizer together with the ETC mechanism, in which nonuniform quantization can enhance the quantization signal-to-noise ratio of the small signal and the key information, which is extracted in the small signal, can be ignored easily [31-33].

The core of this paper is dedicated to consider the circle formation problem of MASs under the constraints of communication bandwidth and limited energy as close to reality as possible. Here, we focus on the special case that each agent can only perceive the angular distance from itself to the nearest neighbor in the counterclockwise direction, while the counterpart in the clockwise direction be acquired via the digital communication network. The main contributions of this paper mainly are threefold. First, a novel algorithm is proposed to tackle the circle formation problem of MASs, in which the nonuniform dynamic quantizer plays a crucial role in data compression and transmission. Second, a distributed event-triggered condition that only relies on the local information of neighboring agents is constructed. Finally, the proof that the designed nonuniform dynamic quantizers will never be saturated is strictly provided, and the comprehensive comparison of advantages and disadvantages of uniform and nonuniform quantizer is shown in detail. In summary, the algorithm proposed in this paper may be favorable to reveal the practical constraints that originated from physical control systems.

An outline of this paper is organised as follows. In Section 2, some basic background and problem statements are given. In Section 3, a new encoder-decoder nonuniform quantizer is designed and the ETC conditions are proposed. Section 4 analyzes the convergence of the proposed control law and proves that the nonuniform quantizer is always in an unsaturated state. Section 5 verifies the feasibility and superiority of the new algorithm through comparative analysis. The conclusion of this article and the prospect of subsequent research directions are referred in Section 6.

\section{Preliminaries and Problem Statement}

In this section, some symbols and basic concepts of algebraic graph theory are collected together. Then, the definition of circle formation and its related properties is mentioned.

2.1. Preliminaries. $\mathbb{R}, \mathbb{R}_{>0}, \mathbb{R}_{\geq 0}$, and $\mathbb{N}$ denote the sets of real, positive real, nonnegative real, and positive integers, respectively. $\mathscr{A}^{T},\|\mathscr{A}\|$, and $\|\mathscr{A}\|_{\infty}$ denote the transpose, Euclidean norm, and infinite norm for a vector or matrix $\mathscr{A}$, respectively. The set of all real matrices with $m$ rows and $n$ columns is denoted as $\mathbb{R}^{m \times n}$. For an arbitrary vector $x, x_{\geq 0}$ means that each element in the vector $x$ is nonnegative. $N$ dimensional column vector whose elements are 1 and 0 is denoted as $1_{N}$ and $0_{N}$, respectively. $I_{N}$ is defined as an $N$ dimensional identity matrix. Given a positive number $z,\lfloor z\rfloor$ represents the integer round-down of $x . \otimes$ represents the Kronecker product, which has the following properties:

(1) $(A \otimes B)(C \otimes D)=(A C) \otimes(B D)$

(2) $(A \otimes B)^{T}=A^{T} \otimes B^{T}$

For a weighted directed graph $\mathscr{G}=\left(\mathscr{V}, \mathscr{E}_{\mathscr{G}}, \mathscr{A}_{\mathscr{G}}\right)$, it contains a set of vertices $\mathscr{V}=\{1,2, \ldots, N\}$, the edge set 
$\mathscr{C}_{\mathscr{G}} \subseteq \mathscr{V} \times \mathscr{V}$, and weighted adjacency matrix $\mathscr{A}_{\mathscr{G}} \in \mathbb{R}_{\geq 0}^{N \times N}$. Self-edges $(i, i)$ are not allowed, i.e., $(i, i) \notin \mathscr{E}_{\mathscr{g}}$ for all $i \in \mathscr{V}$. For $\mathscr{G}$, the corresponding weighted adjacency matrix $\mathscr{A}_{\mathscr{G}}=$ $\left[a_{i j}\right] \in \mathbb{R}_{\geq 0}^{N \times N}$ is defined element-wise with $a_{i j}>0$ if $(i, j) \in \mathscr{E}_{\mathscr{G}}$ and $a_{i j}=0$ otherwise. If there exists an edge $(i, j) \in \mathscr{E}_{\mathscr{G}}$, then we refer to $j$ as an out-neighbor of $i$ and $i$ as an in-neighbor of $j$. For a given node $i$, the sets of out- and in-neighbors are denoted by $N_{i}^{+}$and $N_{i}^{-}$, respectively. A directed path from vertex $i$ to $j$ in a digraph is an ordered sequence of edges starting with $i$ and ending with $j$. If there exists a directed path for any two distinct vertices $i$ and $j$ in $\mathscr{G}$, the digraph $\mathscr{G}$ is called strongly connected. Accordingly, both the out- and in-degree matrices $\mathscr{D}^{+}=\left[d_{1}^{+}, d_{2}^{+}, \ldots, d_{N}^{+}\right]$ and $\mathscr{D}^{-}=\left[d_{1}^{-}, d_{2}^{-}, \ldots, d_{N}^{-}\right]$are diagonal matrices, where $d_{i}^{+}(t)=\sum_{j \in N_{i}^{+}}^{N} a_{i j}$ and $d_{i}^{-}(t)=\sum_{j \in N_{i}^{-}}^{N} a_{i j}$. A digraph is called weight-unbalanced if $\mathscr{D}^{+} \neq \mathscr{D}^{-}$, and the degree of $\mathscr{G}$ is defined as $d^{*}=\max _{i \in \mathscr{V}} d_{i}^{+}$. The Laplacian matrix is defined as $\mathscr{L}=\mathscr{D}^{+}-\mathscr{A}$, which is given in this paper.

Lemma 1 (see [4]). For a weighted directed graph $\mathscr{G}$, there exists the following properties:

(1) The Laplace matrix $\mathscr{L}$ has a zero eigenvalue with associated eigenvector $1_{N}$.

(2) If the graph $\mathscr{G}$ contains a spanning tree, the algebraic multiplicity of eigenvalue zero is simple, and the rest of eigenvalues have positive real parts.

(3) If graph $\mathscr{G}$ is strongly connected, then its Laplacian matrix $\mathscr{L}$ is irreducible and satisfies $\mathscr{L} 1_{N}=0_{N}$. And, there exists a vector $\xi=\left[\xi_{1}, \xi_{2}, \ldots, \xi_{N}\right]^{T}>0$ satisfying $\xi^{T} \mathscr{L}=0_{N}^{T}$ and $\xi^{T} 1_{N}=1$.

Lemma 2 (see [13]). If the unbalanced directed graph $\mathscr{G}$ is strongly connected and defines $\rho_{\lambda}=\max _{2 \leq i \leq N\left|1-h \lambda_{i}\right|}$, $h \in\left(0,1 / d^{*}\right)$, then the Laplacian matrix $\mathscr{L}$ can be decomposed into $\mathscr{L}=\mathscr{T}^{*}$, where $\mathscr{T}^{*}=\left[\xi, \phi_{2}, \ldots, \phi_{N}\right] \in \mathbb{R}^{N \times N}$ and $\mathscr{T}=\left[1_{N}, v_{2}, \ldots, v_{N}\right] \in \mathbb{R}^{N \times N}$ are both nonsingular matrices. $\mathscr{M} \in \mathbb{R}^{N \times N}$ is the matrix $\mathscr{L}$ corresponding to the Jordan standard shape, and its first diagonal element is zero. A variable $\rho(\zeta, h)=\rho_{1}(h) / \zeta, \zeta \in\left(\rho_{\lambda}, 1\right)$, is defined, where $\rho_{1}(h)$ represents the matrix $I_{N}-h \mathscr{M}$. A submatrix is removed from the first row and the first column, and existing real numbers are as follows:

(1) $\mathscr{M}_{\lambda^{\prime}}(\xi, h)=\max _{l \in N}\left\|\rho^{l}(\xi, h)\right\|$

(2) $\mathscr{M}_{\lambda}(\xi, h)=\lim _{l \longrightarrow \infty} \sum_{m}^{l}\left\|\rho^{l}(\xi, h)\right\|$

2.2. Problem Statement. Consider a system composed of $N$ agents, $N \geq 2$, in which each agent is an independent individual with autonomous ability and moves along a preset circle. Initially, all agents are randomly located in different positions on the circle. Here, for the ease of analysis, we marked the agents as 1 to $N$ in the counterclockwise direction, denoted as $x=\left[x_{1}, x_{2}, \ldots, x_{N}\right]^{T} \in \mathbb{R}^{\mathbb{T}}$, as shown in Figure 1. In a fixed coordinate system, the state of the angular position of the agent $i$ at time $t$ is denoted by $x_{i}(t)$. Then, the initial positions of all agents are set to satisfy

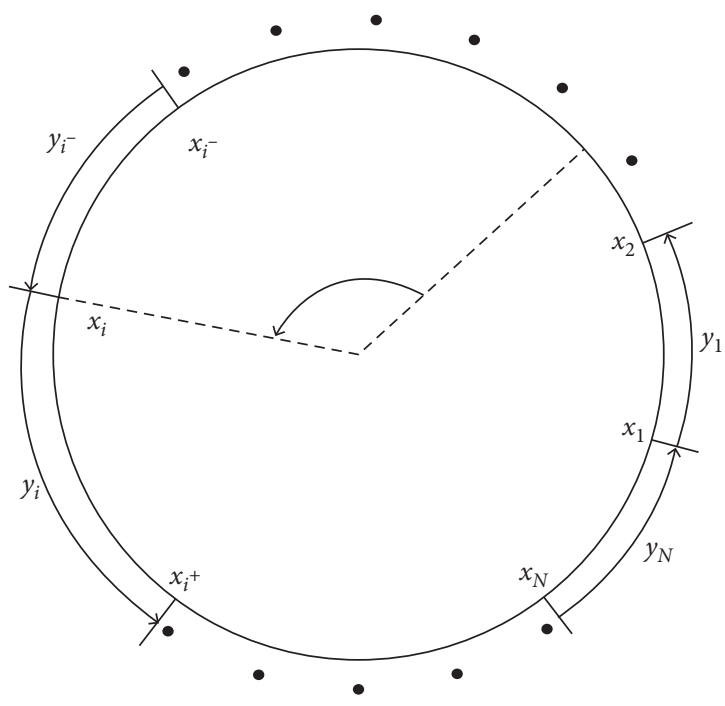

FIgURE 1: Mobile agents located on a circle.

$$
0 \leq x_{1}(0) \leq \cdots \leq x_{i}(0) \leq x_{i+1}(0) \leq \cdots \leq x_{N}(0) \leq 2 \pi .
$$

In this paper, we mainly focus on the case that each agent only has two neighbors that are immediately in front of or behind itself. We denote the set of agent $i$ 's two neighbors by $N_{i}=\left[i^{+}, i^{-}\right]$, where

$$
\begin{aligned}
i^{+} & = \begin{cases}i+1, & i=1,2, \ldots, N-1, \\
1, & i=N,\end{cases} \\
i^{-} & = \begin{cases}N, & i=1, \\
i-1, & i=2,3, \ldots, N .\end{cases}
\end{aligned}
$$

Denote $d_{i} \in \mathbb{R}$ as the desired angular distance between individual $i$ and its neighbor $i^{+}$. The information exchange relationship between agents is further established in such a network, where each agent $i$ (equipped with a unidirectional sensor) can only perceive an angular distance from $i$ to $i^{+}$ and from $i$ to $i^{-}$. The counterpart is obtained through the shared communication network. In this setting, the communication network between agents can be described by a weight-unbalanced directed graph $\mathscr{G}=\left(\mathscr{V}, \mathscr{C}_{\mathscr{G}}, \mathscr{A}_{\mathscr{G}}\right)$, where $\mathscr{V}=\{1,2, \ldots, N\}, \mathscr{C}_{\mathscr{G}}=\{(1,2),(2,3), \ldots,(N-1, N)\}$, and $\mathscr{A}_{\mathscr{G}}=\left[a_{i j}\right] \in \mathbb{R}_{\geq 0}^{N \times N}$, as shown in Figure 2 .

We consider the network of $N$ agents with the dynamics

$x_{i}(t+1)=x_{i}(t)+h u_{i}(t), \quad t=0,1,2, \ldots ; i=1,2, \ldots, N$,

where $x_{i}(t) \in \mathbb{R}$ is the scalar state and $u_{i}(t) \in \mathbb{R}$ is the control input of the agent $i$. Let $y_{i}(t) \in(0,2 \pi), i \in\{1,2, \ldots, N\}$, denote the actual angular distance between the agent $i$ and its counterclockwise neighboring agent $i^{+}$. Thus,

$$
y_{i}(t)= \begin{cases}x_{i^{+}}(t)-x_{i}(t) & i=1,2, \ldots, N-1, \\ x_{i^{+}}(t)-x_{i}(t)+2 \pi & i=N .\end{cases}
$$

Stack vector $y(t)=\left[y_{1}(t), y_{2}(t), \ldots, y_{N}(t)\right]^{T} \in \mathbb{R}^{\mathbb{T}}$. Note that $y_{i}(t)$ is local information that can be acquired by a 


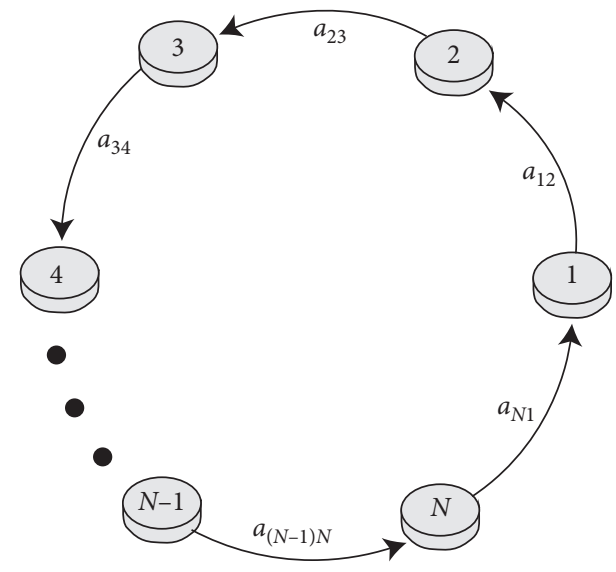

Figure 2: A weight-unbalanced digraph $\mathscr{G}$ with $N$ agents.

unidirectional sensor equipped with agent $i$. In addition, $\sum_{i=1}^{N} y_{i}(t) \equiv 2 \pi$ is always satisfied. The desired circle formation of the MAS is determined by the vector $d=\left[d_{1}, d_{2}, \ldots, d_{N}\right]^{T} \in \mathbb{R}^{\mathbb{T}}$, where $d_{i}$ represents the required angular distance between agents $i$ and $i^{+}$. If $d$ satisfies $d_{i}>0, \forall i \in \mathscr{V}$, and $\sum_{i=1}^{N} d_{i}=2 \pi$, it means that the formation of this desired circle is acceptable.

Now, we are ready to draft the definition of the circle formation problem.

Definition 1 (circle formation problem, see [9]). Given an admissible circle formation characterized by $d \in \mathbb{R}_{\geq 0}^{\mathbb{N}}$, design distributed control laws $u_{i}(t) \in \mathbb{R}, i=1,2, \ldots, N$, such that, under any initial condition (1), the solution to system (2) converges to some equilibrium point $x^{*}$, which satisfies $y^{*}=d$.

Based on the interaction between agents, our main goal in this paper is to explore a meaningful hybrid design nonuniform between quantization techniques and eventtriggered mechanisms to solve the circle formation problems with limited communication bandwidth and limited energy:

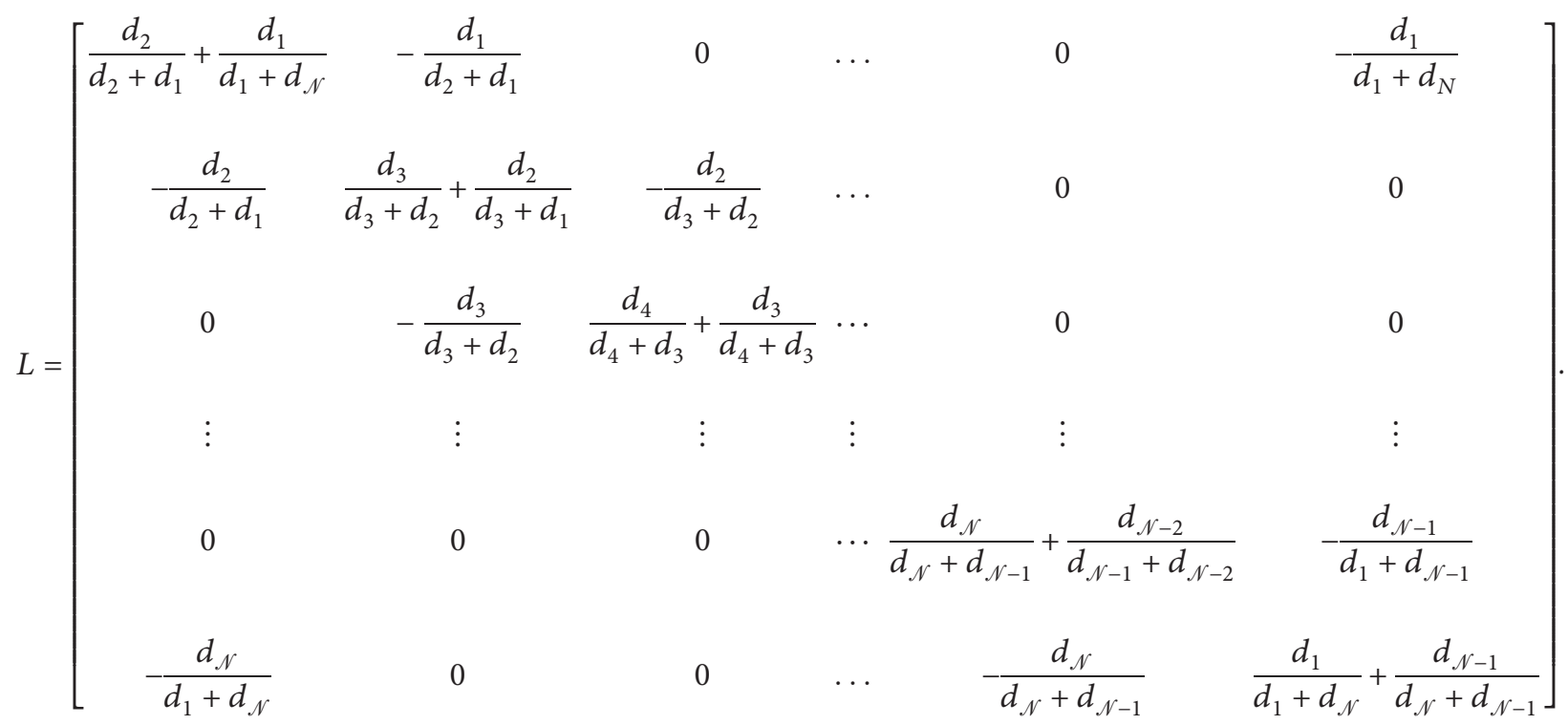

\section{Distributed Control Law Design}

With reference to the communication protocol based on sampling data proposed in [4-6, 9], the following definition is obtained:

$$
u_{i}(t)=\frac{d_{i^{-}}}{d_{i}+d_{i^{-}}} y_{i}(t)-\frac{d_{i}}{d_{i}+d_{i^{-}}} y_{i^{-}}(t), \quad t=0,1,2, \ldots ; i=1,2, \ldots, N
$$

where $i^{-}$represents the neighbor of agent $i$. In (6), for the agent $i$, the fulfillment of the control input requires the exact state information of the agent $i^{-}$. However, the case considered in this paper is that the sensor is unidirectional, and we assume that exact information on the angular distance from the agent $i$ to $i^{-}$is unavailable. To this end, the agents in each pair $\left(i, i^{-}\right)$need to use digital communication channels to exchange information. As described in [4], compared with 
analog signals, digital signals show many advantages, such as signal security, robustness, strength, and less noise interference. Therefore, before transmission, the actual value of the corresponding number of agents $i^{-}$should be quantified. The quantitative communication scheme between agents $i$ and $i^{-}$consists of a dynamic encoder-decoder pair. Then, the corresponding information exchange can be regarded as such a process, at each time step, the relevant state value of the sender is first encoded into symbol data and then transmitted. After receiving the data, the receiver will activate the decoder to obtain an estimate of the relevant state value of the sender.

Following the design of distributed control law (6), some intermediate variables need to be introduced. Define $\delta_{j}(t)=$ $y_{j}(t) / d_{j}$ and $\widehat{\delta}_{j}(t)=\hat{y}_{j}(t) / d_{j}=\xi_{j}\left(t_{k}^{j}\right)$, where $\xi_{j}\left(t_{k}^{j}\right)$ represents the sample value of agent $j$ at $t_{k}^{j}$ th event instant, $j \in \mathscr{V}$. It is worth pointing out that variable conversion will not affect the design of the encoder to the decoder nor will it affect the system stability analysis of the entire encoder.

Next, in a sense, a collaborative design idea is adopted, which is to propose an event-triggered law of nonuniform quantization algorithms combined to solve the problem of circle formation. For each agent $j=\{1,2, \ldots, N\}$, the corresponding event-triggered encoder $\Phi_{j}$ is designed as follows:

$$
\left\{\begin{array}{l}
\xi_{j}(0)=0 \\
\xi_{j}(t)=g(t) s_{j}(t)+\xi_{j}\left(t_{k}^{j}\right) \\
s_{j}(t)=q_{t}\left(\frac{1}{g(t)}\left(x_{j}(t)-\xi_{j}\left(t_{k}^{j}\right)\right)\right)
\end{array}\right.
$$

We use $q_{t}(z)$ to represent a nonuniform quantizer with a finite number of levels. Its role is to map the state deviation value of the agent to the discrete level value of the quantizer. Let $z=\left[z_{1}, z_{2}, \ldots, z_{N}\right]^{T} \in \mathbb{R}^{T}$ and $Q(z)=\left[q_{t}(1)\right.$, $\left.q_{t}(2), \ldots, q_{N}(z)\right]^{T} \in \mathbb{R}^{T}$.

$e_{q i}=\left(s_{j}(t)-(1 / g(t))\left(x_{j}(t)-t \xi_{j} n\left(t_{k}^{j}\right)\right)\right) \quad$ denotes the quantization error. $q_{t}(z)$ can be described as

$$
q_{t}(z)= \begin{cases}0, & e^{-(1 / 2) \beta}-1<z<e^{(1 / 2) \beta}-1, e^{((2 k-1) / 2) \beta}-1 \leq z<e^{((2 k+1) / 2) \beta}-1, \\ k \beta, & k \in\{1,2, \ldots, \Omega\}, \\ \Omega \beta, & z \geq e^{(\Omega+(1 / 2) \beta)}-1, \\ -q_{t}(-z) & z \leq e^{-(1 / 2) \beta}-1,\end{cases}
$$

where $\beta>0$ is the quantization interval and $\Omega$ is the number of quantization level. In each iteration, the communication channel $(i, j) \in \mathscr{E}_{\mathscr{G}}, i \neq j$, is required to have ability of transmitting $\log _{2}(2 \Omega+1)$ bits. If the conditions $z \leq e^{(\Omega+(1 / 2) \beta)}-1$ and $\left|q_{t}(z)-z\right| \leq e^{(\Omega+(1 / 2) \beta)}-1$ are satisfied in the process of information interaction between agents, then the quantizer is not saturated.

Further, the neighboring agent $i$ receives $s_{j}(t)$, which will be estimated by the decoder $\Psi_{j i}$ to obtain the state value of agent $j$. There is a fact that the communication channel is considered to have no noise in the encoding-decoding process. $\Psi_{j i}$ is described as

$$
\left\{\begin{array}{l}
\delta_{j i}(0)=0, \\
\delta_{j i}(t)=g(t-1)\left(e^{s_{j}(t)}-1\right)+\delta_{j i}\left(t_{k}^{j}\right),
\end{array}\right.
$$

where $t=1,2, \ldots, N$ and $\delta_{j i}(t) \in \mathbb{R}$ denotes the output of $\Psi_{j i}$. Relying on the information transmission scheme of the dynamic encoder-decoder described above, the distributed event-triggered circle formation control law of agent $i$ is further designed as

$$
u_{i}(t)=\frac{d_{i} d_{i^{-}}}{d_{i}+d_{i^{-}}}\left(\xi_{i}\left(t_{k}^{i}\right)-\delta_{i^{-}}\left(t_{k}^{i^{-}}\right)\right) .
$$

In above formula (10), $i^{-}$represents the counterclockwise neighbor of the agent $i$ in the clockwise direction. The latest update time and the next update time of the agent $i$ are, respectively, represented as $t_{k}^{i}$ and $t_{k+1}^{i}, t \in\left[t_{k}^{i}, t_{k+1}^{i}\right)$.

Remark 1. It can be observed from encoder-decoder pair (7) and (9) that the advantages are as follows:

(1) At zero initial conditions, the encoder $\Phi_{j}$ and decoder $\Psi_{j i}$ meet the condition $\Phi_{j}=\Psi_{j i}$, which can ensure that both sender and receiver sides have the same estimate of each sender's state.

(2) The term $\delta_{j}(t)-\xi_{j}\left(t_{k}^{j}\right)$ is quantified rather than the state $\delta_{j}(t)$, which can save communication bits and enhance the communication robustness.

(3) The aperiodic event-triggered state $\delta_{j}\left(t_{k}^{j}\right)$ is used to construct the encoder-decoder pair. Compared with the periodic event-triggered, the aperiodic eventtriggered only needs to store the key information when triggering the event conditions rather than each period, and it can save memory for the device equipped with each agent.

Remark 2. In the process of designing the dynamic nonuniform encoder-decoder pair, a scaling function $g(t)$ that has a monotonously decreasing characteristic and attenuates to 0 as $t$ approaches $\infty$ is introduced. It enables the quantizer to be continuously stimulated to strengthen the information 
interaction between agents. At the same time, $g(t)$ should be large enough to ensure that the quantizer is never saturated.

Remark 3. Uniform quantization is also realized by encoder-decoder pairs and an integral way to quantize information in a uniform and fixed interval, which allows both large and small signals to have the same signal-to-noise ratio [34]. However, nonuniform quantization is to quantify the signal in the nonuniform and unfixed interval through its unique compression and spread characteristics, which can improve the signal-to-noise ratio of small signals and thus interpret the information contained in small signals, especially when the event-triggered control strategy is adopted, the event will not be triggered when the signal is small, so it will be ignored, which leads to a large final error of the system. Nonuniform quantization can effectively avoid this problem and improve the consistency of the system.

Then, substituting the protocol (6)-(9) into system (3) and noting (10), we obtain the following closed-loop system:

$$
\begin{aligned}
x_{i}(t+1) & =x_{i}(t)+h u_{i}(t) \\
& =x_{i}(t)+h\left[\sum_{j \in N_{i}^{+}}^{N} a_{i j} \widehat{x}_{j}\left(t_{k j}^{j}\right)-\sum_{j \in N_{i}^{-}}^{N} a_{i j} \widehat{x}_{i}\left(t_{k i}^{i}\right)\right] \\
& =x_{i}(t)+h\left[\sum_{j \in N_{i}^{+}}^{N} a_{i j}\left(\hat{x}_{j}\left(t_{k j}^{j}\right)-\hat{x}_{i}\left(t_{k i}^{i}\right)\right)\right] .
\end{aligned}
$$

Assume that the following conditions exist:

$$
\begin{aligned}
h a_{i i} & =1-\sum_{j=1, j \neq i}^{N} h a_{i j}, \\
\omega_{i j} & =h a_{i j}, \\
\hat{e}_{i}(t) & =\widehat{x}_{i}\left(t_{k i}^{i}\right)-x_{i}(t) .
\end{aligned}
$$

System (11) can be simplified as

$$
\begin{aligned}
x_{i}(t+1) & =x_{i}(t)+h \sum_{j=1}^{N} a_{i j} \widehat{x}_{j}\left(t_{k j}^{j}\right)-\widehat{x}_{i}\left(t_{k i}^{i}\right) \\
& =-\widehat{e}_{i}(t)+\sum_{j=1}^{N} w_{i j} \widehat{x}_{j}\left(t_{k j}^{j}\right) .
\end{aligned}
$$

Denote

$$
\begin{aligned}
X(t) & =\left[x_{1}(t), x_{2}(t), \ldots, x_{n}(t)\right]^{T} \in \mathbb{R}^{N n \times 1}, \\
\widehat{X}(t) & =\left[\widehat{x}_{1}(t), \widehat{x}_{2}(t), \ldots, \widehat{x}_{n}(t)\right]^{T} \in \mathbb{R}^{N n \times 1}, \\
\widehat{e}(t) & =\left[\widehat{e}_{1}(t), \widehat{e}_{2}(t), \ldots, \widehat{e}_{n}(t)\right]^{T} \in \mathbb{R}^{N n \times 1}, \\
\theta(t) & =X(t)-\vartheta_{N} X(t) \in \mathbb{R}^{N n \times 1} .
\end{aligned}
$$

\section{Convergence Analysis}

In this section, we need to prove the issues raised in this paper. Towards this end, some assumptions need to be clearly made at the start.

Assumption 1. $M_{x} \leq \max \left\|x_{i}(0)\right\|$ and $M_{\theta} \leq \max \left\|\theta_{i}(0)\right\|$, where $M_{x}$ and $M_{\theta}$ are known nonnegative constants. For the reason that each agent is defined to accept symbol data only from its neighbors, event-triggered calculations depend only on the local information available to each agent. We propose that the following distributed sampling event-triggered condition for the $i$ th agent satisfies

$$
\widehat{e}_{i}^{2}(t) \geq \sum_{j=1, j \neq i}^{N} w_{i j} \alpha_{i}\left(w_{i i}-\alpha_{i}\right)\left(\hat{x}_{j}\left(t_{k j}^{j}\right)-\widehat{x}_{i}\left(t_{k i}^{i}\right)\right)^{2} .
$$

Remark 4. Zeno behavior generally refers to event-triggered control, where the control is triggered infinitely within a limited time. If Zeno behavior occurs in the system, it will cause a certain degree of delay to the system, which will result in poor system stability and even system hardware failure. It is necessary to strictly consider avoiding Zeno behavior in a continuous-time ETC system, but not in a discrete counterpart. Because there is at least one time step between two consecutive samples in a discrete-time system, Zeno behavior does not occur.

Theorem 1. Based on the condition of strongly connected weight-unbalanced digraph $\mathscr{G}$, combining of the system (2), encoder-decoder pair (4) and (6), and the designed control law (7) as well as the event-triggered condition (11) is achieved. The circle formation problem can be realized when the assumption is valid and the numbers of quantization levels are satisfied.

Proof. A Lyapunov function candidate can be taken as

$$
V=\sum_{i=1}^{N} x_{i}^{2}(t)
$$

Then,

$$
\begin{aligned}
\Delta V= & V(t+1)-V(t) \\
= & \sum_{i=1}^{N} x_{i}^{2}(t+1)-\sum_{i=1}^{N} x_{i}^{2}(t) \\
= & \sum_{i=1}^{N}\left[\hat{e}_{i}^{2}(t)-2 \sum_{j=1}^{N} \widehat{e}_{i}(t) w_{i j} \widehat{x}_{j}\left(t_{k j}^{j}\right)+\left(\sum_{j=1}^{N} w_{i j} \widehat{x}_{j}\left(t_{k j}^{j}\right)\right)^{2}\right] \\
& -\sum_{i=1}^{N} \hat{x}_{i}^{2}(t) .
\end{aligned}
$$




$$
\begin{aligned}
& \text { Since } \\
& \begin{array}{l}
\sum_{j=1}^{N} w_{i j} \widehat{e}_{i}(t) \widehat{x}_{j}\left(t_{k j}^{j}\right) \\
=\sum_{j=1, j \neq i}^{N} w_{i j} \widehat{e}_{i}(t) \widehat{x}_{j}\left(t_{k j}^{j}\right) \\
+\left(1-\sum_{j=1, j \neq i}^{N} w_{i j}\right) \widehat{e}_{i}(t) \widehat{x}_{i}\left(t_{k i}^{i}\right) \\
=\sum_{j=1, j \neq i}^{N} w_{i j} \widehat{e}_{i}(t)\left(\widehat{x}_{j}\left(t_{k j}^{j}\right)-\widehat{x}_{i}\left(t_{k i}^{i}\right)\right) \\
\quad-\widehat{e}_{i}(t) \widehat{x}_{i}\left(t_{k i}^{i}\right)\left(\sum_{j=1}^{N} w_{i j} \widehat{x}_{j}\left(t_{k j}^{j}\right)\right)^{2} \\
=\sum_{j=1}^{N} w_{i j}^{2} \widehat{x}_{j}^{2}\left(t_{k j}^{j}\right)+2 \sum_{j=1}^{N} \sum_{r<j}^{N} w_{i j} w_{i r} \widehat{x}_{j}\left(t_{k j}^{j}\right) \widehat{x}_{r}\left(t_{k r}^{r}\right),
\end{array}
\end{aligned}
$$

we have

$$
\begin{aligned}
& \Delta V=\sum_{i=1}^{N}\left[\sum_{j=1}^{N} w_{i j}^{2} \hat{x}_{j}^{2}\left(t_{k j}^{j}\right)+2 \sum_{j=1}^{N} \sum_{r<j}^{N} w_{i j} w_{i r} \hat{x}_{j}\left(t_{k j}^{j}\right) \hat{x}_{r}\left(t_{k r}^{r}\right)\right] \\
& -2 \sum_{i=1}^{N} \sum_{j=1, j \neq i}^{N} w_{i j} \hat{e}_{i}(t)\left(\hat{x}_{j}\left(t_{k j}^{j}\right)-\hat{x}_{i}\left(t_{k i}^{i}\right)\right) \\
& +\sum_{i=1}^{N} \widehat{e}_{i}^{2}(t)-\widehat{x}_{i}^{2}(t)+2 \widehat{e}_{i}(t) \widehat{x}_{i}\left(t_{k j}^{j}\right) \\
& =\sum_{i=1}^{N}\left[\sum_{j=1}^{N} w_{i j}^{2} \hat{x}_{j}^{2}\left(t_{i j}^{j}\right)+\sum_{j=1}^{N} \sum_{r<j}^{N} w_{i j} w_{i r}\left(\hat{x}_{j}^{2}\left(t_{k j}^{j}\right)+\hat{x}_{r}^{2}\left(t_{k r}^{r}\right)\right)\right] \\
& +\sum_{i=1}^{N} \sum_{j=1}^{N} \sum_{r<j}^{N} w_{i j} w_{i r}\left(-\widehat{x}_{j}^{2}\left(t_{k j}^{j}\right)\right) \\
& \left.-\widehat{x}_{r}^{2}\left(t_{k r}^{r}\right)+2 \widehat{x}_{j}\left(t_{k j}^{j}\right) \widehat{x}_{r}\left(t_{k r}^{r}\right)\right) \\
& -2 \sum_{i=1}^{N} \sum_{j=1, j \neq i}^{N} w_{i j} \widehat{e}_{i}(t)\left(\hat{x}_{j}\left(t_{k j}^{j}\right)-\widehat{x}_{i}\left(t_{k i}^{i}\right)\right) \\
& +\sum_{i=1}^{N} \widehat{e}_{i}^{2}(t)-\widehat{x}_{i}^{2}(t)+2 \widehat{e}_{i}(t) \widehat{x}_{i}\left(t_{k i}^{i}\right) .
\end{aligned}
$$

Note that

$$
\begin{aligned}
& \sum_{i=1}^{N}\left[\sum_{j=1}^{N} w_{i j}^{2} \hat{x}_{j}^{2}\left(t_{k j}^{j}\right)+\sum_{j=1}^{N} \sum_{r<j}^{N} w_{i j} w_{i r}\left(\hat{x}_{j}^{2}\left(t_{k j}^{j}\right)+\hat{x}_{r}^{2}\left(t_{k r}^{r}\right)\right)\right] \\
& =\sum_{i=1}^{N}\left[\sum_{j=1}^{N} w_{i j}^{2} \hat{x}_{j}^{2}\left(t_{k j}^{j}\right)+\sum_{j=1}^{N} \sum_{r=1, r \neq j}^{N} w_{i j} w_{i r} \widehat{x}_{j}\left(t_{k j}^{j}\right)\right. \\
& =\sum_{i=1}^{N} \sum_{j=1}^{N} \sum_{r=1}^{N} w_{i j} w_{i r} x_{j}^{2}\left(t_{k j}^{j}\right) \\
& =\sum_{i=1}^{N} \hat{x}_{j}^{2}\left(t_{k j}^{j}\right),
\end{aligned}
$$

$$
\begin{aligned}
\sum_{i=1}^{N} \sum_{j=1}^{N} \sum_{r<j}^{N} w_{i j} w_{i r}\left(-\hat{x}_{j}\left(t_{k j}^{j}\right)-\hat{x}_{r}\left(t_{k r}^{r}\right)+2 \hat{x}_{j}\left(t_{k j}^{j}\right) \widehat{x}_{r}\left(t_{k r}^{r}\right)\right) \\
=-\sum_{i=1}^{N} \sum_{j=1}^{N} \sum_{r<j}^{N} w_{i j} w_{i r}\left(\hat{x}_{j}\left(t_{k j}^{j}\right)-\hat{x}_{r}\left(t_{k r}^{r}\right)\right)^{2} \\
=-\sum_{i=1}^{N}\left[\sum_{j=1, j \neq i}^{N} \sum_{r=j, r \neq i}^{N} w_{i j} w_{i r}\left(\hat{x}_{j}\left(t_{k j}^{j}\right)-\hat{x}_{r}\left(t_{k r}^{r}\right)\right)^{2}\right. \\
\left.\quad+\sum_{j=1, j \neq i}^{N} w_{i j} w_{i i}\left(\hat{x}_{j}\left(t_{k j}^{j}\right)-\widehat{x}_{r}\left(t_{k r}^{r}\right)\right)\right] .
\end{aligned}
$$

Under the Young inequality [35], for any $x$ and $y \in \mathbb{R}$ and $\tau \in \mathbb{R}, \quad \tau>0$, it has the following properties $x y \leq(\tau / 2) x^{2}+(1 / 2 \tau) y^{2}$, and we have

$$
\begin{aligned}
& -2 \sum_{i=1}^{N} \sum_{j=1, j \neq i}^{N} w_{i j} \widehat{e}_{i}(t)\left(\hat{x}_{j}\left(t_{k j}^{j}\right)-\widehat{x}_{i}\left(t_{k i}^{i}\right)\right) \\
\leq & \sum_{i=1}^{N} \sum_{j=1, j \neq i}^{N} 2 w_{i j}\left[\frac{\hat{e}_{i}^{2}(t)}{2 \alpha_{i}}+\frac{\alpha_{i}}{2}\left(\hat{x}_{j}\left(t_{k j}^{j}\right)-\widehat{x}_{i}\left(t_{k i}^{i}\right)\right)^{2}\right] \\
\leq & \sum_{i=1}^{N} \sum_{j=1, j \neq i}^{N} w_{i j}\left[\frac{\hat{e}_{i}^{2}(t)}{\alpha_{i}}+\alpha_{i}\left(\hat{x}_{j}\left(t_{k j}^{j}\right)-\hat{x}_{i}\left(t_{k i}^{i}\right)\right)^{2}\right], \\
& \sum_{i=1}^{N}\left(\hat{e}_{i}^{2}(t)-\widehat{x}_{i}^{2}(t)-2 \widehat{e}_{i}(t) \widehat{x}_{i}\left(t_{k i}^{i}\right)\right) \\
= & \sum_{i=1}^{N}\left[\widehat{e}_{i}^{2}(t)-\left(\hat{x}_{i}\left(t_{k i}^{i}\right)-\widehat{e}_{i}(t)\right)^{2}-2 \widehat{e}_{i}(t) \widehat{x}_{i}\left(t_{k i}^{i}\right)\right] \\
= & -\sum_{i=1}^{N} \widehat{x}_{i}^{2}\left(t_{k i}^{i}\right) .
\end{aligned}
$$

Combining (20)-(23), we can obtain 


$$
\begin{aligned}
\Delta V \leq & \sum_{j=1}^{N} \hat{x}_{j}^{2}\left(t_{k j}^{j}\right)-\sum_{i=1}^{N}\left[\sum_{j=1, j \neq i}^{N} \sum_{k=j, r \neq i}^{N} w_{i j} w_{i r}\left(\hat{x}_{j}\left(t_{k j}^{j}\right)-\hat{x}_{r}\left(t_{k r}^{r}\right)\right)^{2}\right. \\
& \left.+\sum_{j=1, j \neq i}^{N} w_{i j} w_{i i}\left(\hat{x}_{j}\left(t_{k j}^{j}\right)-\hat{x}_{r}\left(t_{k r}^{r}\right)\right)\right] \\
& -\sum_{i=1}^{N} \sum_{j=1, j \neq i}^{N} w_{i j}\left(\frac{\hat{e}_{i}^{2}(t)}{\alpha_{i}}+\alpha_{i}\left(\hat{x}_{j}\left(t_{k j}^{j}\right)-\hat{x}_{i}\left(t_{k i}^{i}\right)\right)^{2}\right)-\sum_{i=1}^{N} \hat{x}_{i}^{2}\left(t_{k i}^{i}\right) \\
\leq & \sum_{i=1}^{N} \sum_{j=1, j \neq i}^{N} w_{i j}\left[\frac{\hat{e}_{i}^{2}(t)}{\alpha_{i}}-\left(w_{i i}-\alpha_{i}\right)\left(\hat{x}_{j}\left(t_{k j}^{i}\right)-\hat{x}_{i}\left(t_{k i}^{i}\right)\right)^{2}\right] \\
\leq & \sum_{i=1}^{N}\left[\frac{\hat{e}_{i}^{2}(t)}{\alpha_{i}}-\sum_{j=1, j \neq i}^{N} w_{i j}\left(w_{i i}-\alpha_{i}\right)\left(\hat{x}_{j}\left(t_{k j}^{j}\right)-\hat{x}_{i}\left(t_{k t}^{i}\right)\right)^{2}\right] .
\end{aligned}
$$

Based on the event-triggered conditions (15) given above, we can obtain that the system is stable when $\widehat{e}_{i}(t)$ meets the following conditions:

$$
\widehat{e}_{i}^{2}(t)<\sum_{j=1, j \neq i}^{N} w_{i j} \alpha_{i}\left(w_{i i}-\alpha_{i}\right)\left(\hat{x}_{j}\left(t_{k j}^{j}\right)-\widehat{x}_{i}\left(t_{k i}^{i}\right)\right)^{2} .
$$

Theorem 2. If Assumption 1 is satisfied, given any admissible circle formation characterized by $d$, consider the system in (3) with the combination between dynamic encoder-decoder pair (7) and (9), designed control law (10), and scaling function $s_{j}(t)-s_{0} \xi$ over a strongly connected weight-unbalanced digraph $\mathscr{G}$. For given $h \in\left(0,1 / d^{*}\right)$ and $\xi \in\left(\rho_{\lambda}, 1\right)$, the solvability of the circle formation problem is achieved when the upper bound of quantization $\mathscr{H}_{1}(\xi, h)$ satisfies

$$
\mathscr{H}_{1}(\xi, h)=\left\lfloor E(\xi, h)-\frac{1}{2} \beta\right\rfloor+\beta,
$$

where

$$
E(\xi, h)=\frac{\left(1+2 h d^{*}\right)}{\xi}\left(e^{((2 \Omega+1) / 2) \beta}-1\right)+\frac{2 h^{2} d^{* 2}\|v\|_{\infty}\|\phi\|_{\infty}}{\xi\left(\xi-\rho_{\lambda}\right)},
$$

and the condition

$$
s_{0}>\max \left[\frac{2 M_{x}}{(2 \mathscr{H}-1) \varepsilon}, \frac{\left(\xi-\rho_{\lambda}\right)\left(\xi M_{\theta}+2 h d^{*} M_{x}\right)}{h d^{*}}\right],
$$

holds simultaneously, where $\beta$ represents the quantization interval and $\mathscr{H} \leq\left(\mathscr{H}_{1}(\xi, h) / \beta\right)$. That is to say, system (3) satisfies $\lim _{t \rightarrow \infty} y(t)=d$. Furthermore, all the quantizers will never be saturated.

Proof. Combining equations (7)-(9), we have

$$
\left\{\begin{array}{l}
X(t+1)=\left(\left(I_{N}-h L\right) \otimes I_{n}\right) X(t)-\left(h L \otimes I_{n}\right) \widehat{e}(t), \\
\widehat{X}(t+1)=g(t)\left(e^{q_{t}((1 / g(t))(x(t+1)-\widehat{x}(t)))}+1\right)+\widehat{X}(t) .
\end{array}\right.
$$

According to $\mathscr{J}_{N} L=L \mathscr{J}_{N}=0$, we can get

$$
\begin{aligned}
& X(t+1)-\widehat{X}(t) \\
& =\left(\left(I_{N}-h L\right) \otimes I_{n}\right) X(t)+\left(h L \otimes I_{n}\right) \widehat{e}(t)+g(t)\left(e^{q_{t}((1 / g(t))(X(t+1)-\widehat{X}(t)))}+1\right)+\widehat{X}(t) \\
& =\left(\left(I_{N}-h L\right) \otimes I_{n}\right) \widehat{e}(t)-\left(h L \otimes I_{n}\right)\left(X(t)-J_{N} X(t)\right) \\
& =\left(\left(I_{N}-h L\right) \otimes I_{n}\right) \widehat{e}(t)-\left(h L \otimes I_{n}\right) \theta(t) .
\end{aligned}
$$

Then,

$$
\left\{\begin{array}{l}
\theta(t+1)=\left(\left(I_{N}-h L\right) \otimes I_{n}\right) \theta(t)+\left(h L \otimes I_{n}\right) \widehat{e}(t), \\
\widehat{e}(t+1)=\left(\left(I_{N}-h L\right) \otimes I_{n}\right) \widehat{e}(t)+\left(h L \otimes I_{n}\right) \theta(t)-g(t)\left(e^{q_{t}((1 / g(t))(X(t+1)-\widehat{X}(t)))}+1\right) .
\end{array}\right.
$$


To make it easier to calculate, some new variables are introduced as

$$
\begin{aligned}
& \varphi(t)=\frac{\widehat{e}(t)}{g(t)}, \\
& \mu(t)=\frac{\theta(t)}{g(t)}, \\
& g(t)=s_{0} \xi^{t} .
\end{aligned}
$$

From $s_{j}(t)-s_{0} \xi$ and (31), we can get

$$
\left\{\begin{array}{l}
\mu(t+1)=\xi^{-1}\left(\left(I_{N}-h L\right) \oplus I_{n}\right) \theta(t)+\xi^{-1}\left(h L \otimes I_{n}\right) \varphi(t), \\
\varphi(t+1)=\xi^{-1}\left(\left(I_{N}-h L\right) \oplus I_{n}\right) \varphi(t)-\left(h L \otimes I_{n}\right) \mu(t)-\left(e^{q_{t}((1 / g(t))(X(t+1)-\tilde{X}(t)))}+1\right) .
\end{array}\right.
$$

For a clear expression, we summarize the procedure of proof by dividing into two cases:
Case 1: when $t=0$ and $X(0)=0_{N}$, we get

$$
\begin{gathered}
\|\varphi(0)\|_{\infty}=\left\|\frac{\hat{e}(0)}{s_{0}}\right\|_{\infty} \leq \frac{M_{x}}{s_{0}}, \\
\left\|\left(\left(I_{N}-h L\right) \otimes I_{n}\right) \varphi(0)-\left(h L \otimes I_{n}\right) \mu(0)\right\|_{\infty}=\left\|\frac{X_{0}}{s_{0}}\right\|_{\infty} \leq \frac{M_{x}}{s_{0}} \leq e^{((2 \Omega+1) / 2) \beta}-1 .
\end{gathered}
$$

Obviously, when $t=0$, the quantizer is not saturated.

Case 2: when $t \geq 0$, define a nonnegative integer $r=1,2, \ldots, t$; then, we assume that

$$
\sup _{0<t<r}\left\|\left[\left(\left(I_{N}-h L\right) \otimes I_{n}\right) \varphi(t)-\left(h L \otimes I_{n}\right) \mu(t)-\left(\exp \left(q_{t}\left(\frac{1}{g(t)}\left(\left(I_{N}-h L\right) \otimes I_{n}\right) \varphi(t)-\left(h L \otimes I_{n}\right) u(t)\right)\right)-1\right)\right]\right\|_{\infty} \leq e^{(1 / 2) \beta}-1,
$$

then

$$
\sup _{0 \leq r}\|\varphi(t)\|_{\infty} \leq \frac{1}{\xi}\left(e^{(1 / 2) \beta}-1\right) .
$$

Let $\bar{\mu}(t)=T^{*} \mu(t)$, relying on Lemma 2 $\bar{\mu}(t)=\left[\bar{\mu}_{1}(t), \bar{\mu}_{2}(t)\right]^{T}, \quad \bar{\mu}_{1}(t) \in \mathbb{R}^{1 \times N n}, \quad$ and $\bar{\mu}_{2}(t) \in \mathbb{R}^{(N-1) n \times N n}$. Here, $\bar{\mu}_{1}(t)=0$, which is available:

$$
\begin{aligned}
\bar{\mu}(t+1) & =T^{*} \mu(t+1) \\
& =\xi^{-1}\left(\left(T^{*}-h M T^{*}\right) \otimes I_{n}\right) \mu(t)+\xi^{-1}\left(h M T^{*} \otimes I_{n}\right) \varphi(t) .
\end{aligned}
$$

Let $\quad \phi=\left[\phi_{2}, \ldots, \phi_{N}\right] \in \mathbb{R}^{(N-1) n \times N n} \quad$ and $v=\left[v_{2}, \ldots, v_{N}\right] \in \mathbb{R}^{N n \times(N-1) n}$. Define $\quad t=r+1$, $\bar{\mu}_{2}(t)=\phi \mu(t)$, and $\mu(t)=v \bar{\mu}_{2}(t)$, and formula (40) can be organized as

$$
\mu(r+1)=v \rho^{r+1}(\xi, h) \phi \bar{\mu}_{2}(0)-v \rho^{r}(\xi, h) \xi^{-1}\left(h \phi L \otimes I_{n}\right) \varphi(0)-v \sum_{m=0}^{r-1} \rho^{m}(\xi, h) \xi^{-1}\left(h \phi L \otimes I_{n}\right) \varphi(r-m)
$$

where 


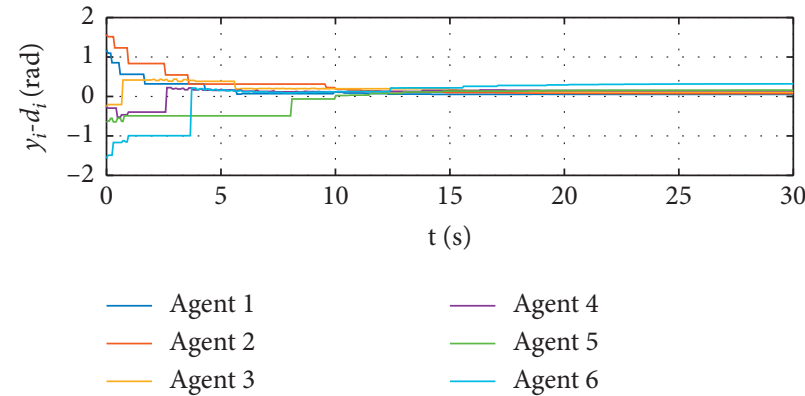

(a)

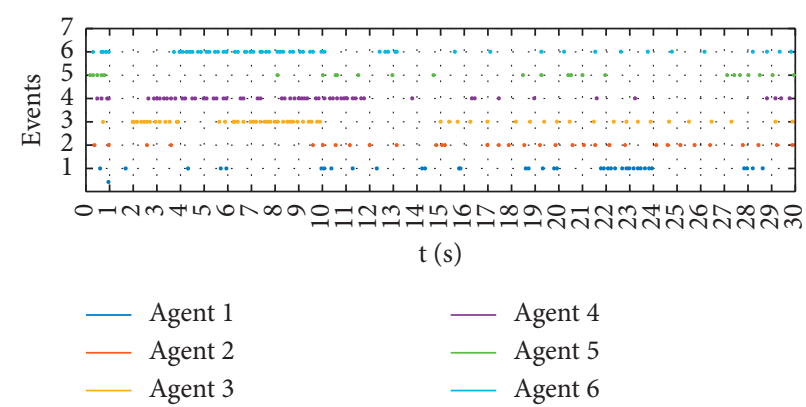

(b)

Figure 3: Simulation results of the uniform quantification algorithm in [9]. (a) Represents the evolution of the difference between current angular distance and the desired one between each pair of neighboring agents. (b) Represents the event sequences of $N=6$ agents.

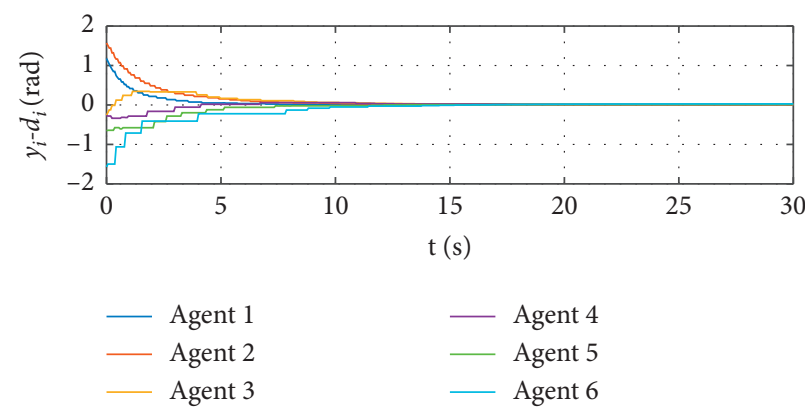

(a)

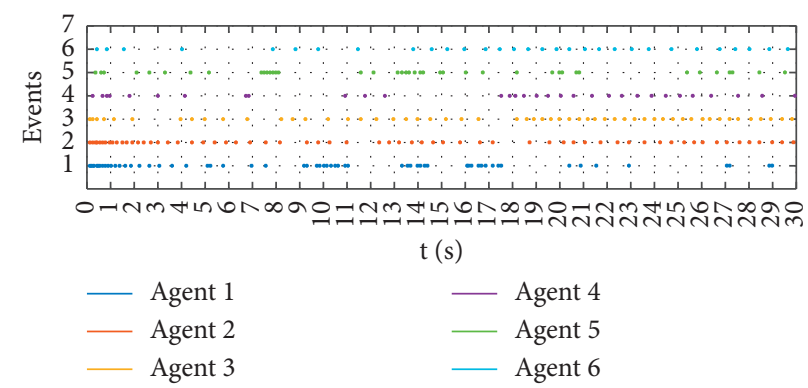

(b)

Figure 4: Simulation results of the nonuniform quantification algorithm. (a) Represents the evolution of the difference between current angular distance and the desired one between each pair of neighboring agents. (b) Represents the event sequences of $N=6$ agents.

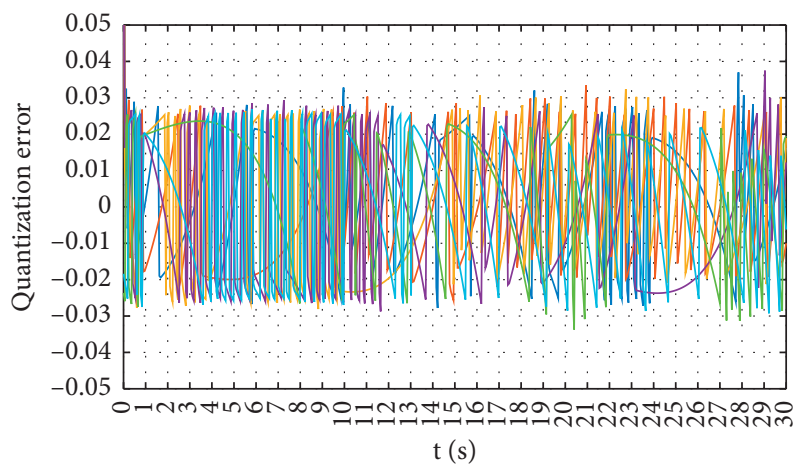

FiguRE 5: Uniform quantization error $e_{q i}(t)(i=1,2, \ldots, 6)$.

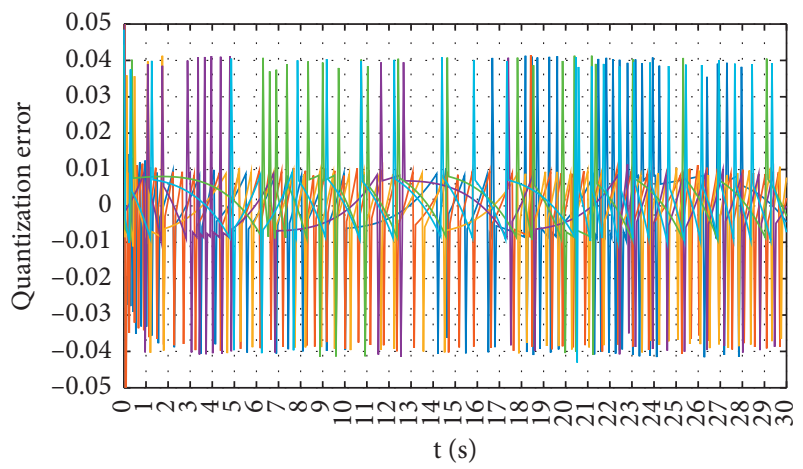

Figure 6: Nonuniform quantization error $e_{q i}(t)(i=1,2, \ldots, 6)$.

$$
\left\|v \rho^{r+1}(\xi, h) \phi \bar{\mu}_{2}(0)\right\|_{\infty} \leq\|v\|_{\infty}\left\|\rho^{r+1}(\xi, h)\right\|_{\infty}\|\phi\|_{\infty}\left\|\bar{\mu}_{2}(0)\right\|_{\infty} \leq \frac{\|v\|_{\infty}\|\phi\|_{\infty} M_{\theta}}{s_{0}}\left(\frac{\rho_{\lambda}}{\xi}\right)^{r} .
$$

Based on $\|L\|_{\infty}=d^{*}, \xi \in\left(\rho_{\lambda}, 1\right)$, and we have 


$$
\begin{aligned}
& \left\|v \rho^{r}(\xi, h) \xi^{-1}\left(h \phi L \otimes I_{n}\right) \varphi(0)\right\|_{\infty} \\
& \leq\|v\|_{\infty}\left\|\rho^{r}(\xi, h)\right\|_{\infty} \xi^{-1} h\|\phi\|_{\infty}\left\|L \otimes I_{n}\right\|_{\infty}\|\varphi(0)\|_{\infty} \\
& \leq \frac{2 h d^{*}\|v\|_{\infty}\|\phi\|_{\infty} M_{\infty}}{s_{0} \xi}\left(\frac{\rho_{\lambda}}{\xi}\right)^{r} . \\
& \left\|v \sum_{m=0}^{r-1} \rho^{m}(\xi, h) \xi^{-1}\left(h \phi L \otimes I_{n}\right) \varphi(r-m)\right\|_{\infty} \leq \frac{2 h d^{*}\|u\|_{\infty}\|\phi\|_{\infty}}{\xi\left(\xi-\rho_{\lambda}\right)}\left(1-\left(\frac{\rho_{\lambda}}{\xi}\right)^{r}\right) .
\end{aligned}
$$

Depending on Theorem 2 and formulas (39)-(41), we have

$$
\begin{aligned}
\|\mu(r+1)\|_{\infty} & \leq \frac{\|v\|_{\infty}\|\phi\|_{\infty} M_{\theta}}{s_{0}}\left(\frac{\rho_{\lambda}}{\xi}\right)^{r}+\frac{2 h d^{*}\|v\|_{\infty}\|\phi\|_{\infty} M_{x}}{s_{0} \xi}\left(\frac{\rho_{\lambda}}{\xi}\right)^{r}+\frac{2 h d^{*}\|v\|_{\infty}\|\phi\|_{\infty}}{\xi\left(\xi-\rho_{\lambda}\right)}\left(1-\left(\frac{\rho_{\lambda}}{\xi}\right)^{r}\right) \\
& \leq \max \left(\frac{\xi\|\boldsymbol{\mu}\|_{\infty}\|\phi\|_{\infty} M_{\theta}+2 h d^{*}\|v\|_{\infty}\|\phi\|_{\infty} M_{x}}{s_{0} \xi} \xi^{*}+\frac{h d^{*}\|v\|_{\infty}\|\phi\|_{\infty}}{\xi\left(\xi-\rho_{\lambda}\right)}\left(1-\xi^{*}\right)\right) .
\end{aligned}
$$

Then,

$$
\begin{aligned}
& \left\|\left(\left(I_{N}-h L\right) \otimes I_{n}\right) \varphi(r-1)-\left(h L \otimes I_{n}\right) \mu(r-1)\right\|_{\infty} \\
& \left.\leq \|\left(I_{N}-h L\right) \otimes I_{n}\right) \varphi(r-1)\left\|_{\infty}+\right\|\left(h L \otimes I_{n}\right) \mu(r-1) \|_{\infty} \\
& \leq \frac{\left(1+2 h d^{*}\right)}{\xi}\left(e^{((2 \Omega+1) / 2) \beta}-1\right)+2 h d^{*} \max \left[\frac{\xi\|v\|_{\infty}\left\|_{\infty}\right\|_{\infty} M_{\theta}+2 h d^{*}\|v\|_{\infty}\left\|_{\infty} M\right\|_{\infty} M_{\infty}}{s_{0} \xi} \xi^{*}+\frac{h d^{*}\|v\|_{\infty}\|\phi\|_{\infty}}{\xi\left(\xi-\rho_{\lambda}\right)}\left(1-\xi^{*}\right)\right] \\
& =\frac{\left(1+2 h d^{*}\right)}{\xi}\left(e^{((2 \Omega+1) / 2) \beta}-1\right)+\frac{2 h^{2} d^{* 2}\|v\|_{\infty}\|\phi\|_{\infty}}{\xi\left(\xi-\rho_{\lambda}\right)} \\
& =E(\xi, h) \leq e^{E(\xi, h)}-1 \leq e^{[E(\xi, h)-(1 / 2) \beta]+(3 / 2) \beta}-1 \\
& =e^{\mathscr{H} 1(\xi, h)+(1 / 2) \beta}-1=e^{((2 \Omega+1) / 2) \beta}-1 .
\end{aligned}
$$

That is to say, all the quantizers are not saturated when the above condition is satisfied by quantizer level number $(2 \Omega-1)$.

\section{Numerical Examples}

In this section, a multiagent system composed of $N=6$ agents is used to simulate and verify the superiority of the nonuniform quantization algorithm. Under the condition that the initial conditions of each agent in the system satisfy (1), $h=0.06 \mathrm{~s}$ is selected as the sampling period of the system. Under the condition that the proposed algorithm can be realized, the expected angular distance between agents is set to $d=[(\pi / 8),(\pi / 6),(\pi / 4),(\pi / 3),(3 \pi / 8)$, $(3 \pi / 4)]$. We set the quantization interval correlation amount $\beta=0.2, s_{0}=10$, and $\xi=0.98288$. The simulation results are shown in the figure.

Comparing the simulation results of uniform quantization algorithm (Figure 3) and nonuniform quantization algorithm (Figure 4) can get some conclusions. Due to the characteristics of nonuniform quantization, a better quantized signal-to-noise ratio can be obtained when processing small signals. The error of nonuniform quantization will be more smaller. In other words, nonuniform quantization can effectively improve the system accuracy. It is obvious that the nonuniform quantization tracking effect is better and the 
final error is significantly reduced when the event-triggered mechanism is considered. The reason why nonuniform quantization triggers less than uniform quantization is that nonuniform quantization can improve the SNR of small signals and trigger events to update the control protocol even when the signal is small. However, uniform quantization tends to ignore this signal, which leads to the accumulation of errors and ultimately increases the frequency of events. In summary, the nonuniform quantization algorithm proposed in this paper can better improve the system accuracy and can improve the system performance under the same conditions.

As shown in Figures 5 and 6, the quantization error of each agent is $e_{q i}(t)=s_{j}(t)-\left((1 / g(t))\left(x_{j}(t)-t \xi_{j} n\left(t_{k}^{j}\right)\right)\right)$. In the nonuniform quantization algorithm, $s_{j}(t)$ is log-scaled, so the quantization error exhibits the phenomenon in Figure 6. It is worth noting that the quantization error of each agent's quantizer is less than its upper limit and saturation will never occur.

\section{Conclusions}

This paper mainly explores the existing uniform quantization method further and designs a new nonuniform quantization algorithm combined with the event-triggered mechanism. First of all, in order to improve the information exchange between agents, we propose a scheme based on nonuniform dynamic codec, which effectively solves the problem of small signal loss during information exchange between agents. Then, a distributed control algorithm based on the coordinated control of the event-triggered mechanism and nonuniform encoder is given to reduce system energy consumption. In addition, all designed quantizers do not appear to be saturated. Finally, numerical simulation results verify the effectiveness of the algorithm. Future work will focus on solving practical problems, such as the rapid convergence of multiagents under limited input and the consistency of general nonlinear systems.

\section{Data Availability}

The raw/processed data required to reproduce these findings cannot be shared at this time as the data also forms part of an ongoing study.

\section{Conflicts of Interest}

The authors declare that they have no conflicts of interest.

\section{Acknowledgments}

This work was supported in part by grants from the National Natural Science Foundation of China (nos. 61963006, 61563005, 61563006, 61973007, and 61633002), Natural Science Foundation of Guangxi Province of China (Grant nos. 2018GXNSFAA050029 and 2018GXNSFAA294085), Innovation Project of Guangxi University of Science and Technology Graduate Education (no. GKYC202006), and Project of Guangxi Key Laboratory of Automobile Components and Vehicle Technology (no. 2017GKLACVTZZ02).

\section{References}

[1] B. D. O. Anderson, Z. Sun, T. Sugie, S.-I. Azuma, and K. Sakurama, "Formation shape control with distance and area constraints," IFAC Journal of Systems and Control, vol. 1, pp. 2-12, 2017.

[2] K.-K. Oh, M.-C. Park, and H.-S. Ahn, "A survey of multiagent formation control," Automatica, vol. 53, pp. 424-440, 2015.

[3] X. Yang, L. Liao, Q. Yang, B. Sun, and J. Xi, "Limited-energy output formation for multiagent systems with intermittent interactions," Journal of the Franklin Institute, 2021.

[4] J. Wen, C. Wang, P. Xu, and G. Xie, "Decentralized eventtriggered circle formation control for multiagent systems via synchronous periodic event detection," International Journal of Robust and Nonlinear Control, vol. 30, no. 3, pp. 910-925, 2020.

[5] C. Wang and G. Xie, "Limit-cycle-based decoupled design of circle formation control with collision avoidance for anonymous agents in a plane," IEEE Transactions on Automatic Control, vol. 62, no. 12, pp. 6560-6567, 2017.

[6] P. Xu, J. Wen, C. Wang, and G. Xie, "Distributed circle formation control over directed networks with communication constraints," IFAC-PapersOnLine, vol. 52, no. 3, pp. 108-113, 2019.

[7] L. Zhang and L. Sun, "Multi-objective service restoration for blackout of distribution system with distributed generators based on multi-agent ga," Energy Procedia, vol. 12, pp. 253262, 2011

[8] W. Liu, S. Zhou, S. Yan, and Q. Wu, "LQR_based consensus algorithms of muti-agent systems with a prescribed convergence speed," in Proceedings of 2014 IEEE Chinese Guidance, Navigation and Control Conference, pp. 868-873, Yantai, China, August 2014.

[9] J. Wen, P. Xu, C. Wang, G. Xie, and Y. Gao, "Distributed event-triggered circle formation control for multi-agent systems with limited communication bandwidth," Neurocomputing, vol. 358, pp. 211-221, 2019.

[10] A. Kashyap, T. Basar, and R. Srikant, "Consensus with quantized information updates," in Proceedings of the 45th IEEE Conference on Decision and Control, pp. 2728-2733, San Diego, CA, USA, December 2006.

[11] P. Frasca, R. Carli, F. Fagnani, and S. Zampieri, "Average consensus on networks with quantized communication," International Journal of Robust and Nonlinear Control, vol. 19, no. 16, pp. 1787-1816, 2009.

[12] R. Carli, F. Fagnani, P. Frasca, and S. Zampieri, "Efficient quantized techniques for consensus algorithms," in Proceedings of the NeCST workshop, pp. 1-8, Nancy, France, June 2007.

[13] H. Li, G. Chen, T. Huang, and Z. Dong, "High-performance consensus control in networked systems with limited bandwidth communication and time-varying directed topologies," IEEE Transactions on Neural Networks and Learning Systems, vol. 28, no. 5, pp. 1043-1054, 2016.

[14] T. Li, M. Fu, L. Xie, and J.-F. Zhang, "Distributed consensus with limited communication data rate," IEEE Transactions on Automatic Control, vol. 56, no. 2, pp. 279-292, 2010.

[15] T.-F. Ding, M.-F. Ge, C.-H. Xiong, and J. H. Park, "Bipartite consensus for networked robotic systems with quantized-data interactions," Information Sciences, vol. 511, pp. 229-242, 2020.

[16] Z. Zeng, X. Wang, Z. Zheng, and L. Zhao, "Edge agreement of second-order multi-agent system with dynamic quantization 
via the directed edge laplacian," Nonlinear Analysis: Hybrid Systems, vol. 23, pp. 1-10, 2017.

[17] J. Li, D. W. C. Ho, and J. Li, "Adaptive consensus of multiagent systems under quantized measurements via the edge laplacian," Automatica, vol. 92, pp. 217-224, 2018.

[18] Z.-M. Li, X.-H. Chang, K. Mathiyalagan, and J. Xiong, "Robust energy-to-peak filtering for discrete-time nonlinear systems with measurement quantization," Signal Processing, vol. 139, pp. 102-109, 2017.

[19] C.-E. Ren, L. Chen, C. L. P. Chen, and T. Du, "Quantized consensus control for second-order multi-agent systems with nonlinear dynamics," Neurocomputing, vol. 175, pp. 529-537, 2016.

[20] Q. Zheng, S. Xu, and Z. Zhang, "Asynchronous nonfragile $H_{\infty}$ filtering for discrete-time nonlinear switched systems with quantization," Nonlinear Analysis: Hybrid Systems, vol. 37, Article ID 100911, 2020.

[21] Z. Xu, C. Li, and Y. Han, "Leader-following fixed-time quantized consensus of multi-agent systems via impulsive control," Journal of the Franklin Institute, vol. 356, no. 1, pp. 441-456, 2019.

[22] G. Guo, L. Ding, and Q.-L. Han, "A distributed event-triggered transmission strategy for sampled-data consensus of multi-agent systems," Automatica, vol. 50, no. 5, pp. 1489-1496, 2014.

[23] L. Hetel, C. Fiter, H. Omran et al., "Recent developments on the stability of systems with aperiodic sampling: an overview," Automatica, vol. 76, pp. 309-335, 2017.

[24] T. Li, H. Zhao, and Y. Chang, "A novel event-triggered communication strategy for second-order multiagent systems," ISA Transactions, vol. 97, pp. 93-101, 2020.

[25] T. Li, Z. Li, S. Fei, and Z. Ding, "Second-order event-triggered adaptive containment control for a class of multi-agent systems," ISA Transactions, vol. 96, pp. 132-142, 2020.

[26] G. Liu, Y. Pan, H.-K. Lam, and H. Liang, "Event-triggered fuzzy adaptive quantized control for nonlinear multi-agent systems in nonaffine pure-feedback form," Fuzzy Sets and Systems, vol. 416, pp. 27-46, 2021.

[27] Y. Xie and Z. Lin, "Event-triggered global stabilization of general linear systems with bounded controls," Automatica, vol. 107, pp. 241-254, 2019.

[28] X. Dong and G. Hu, "Time-varying formation control for general linear multi-agent systems with switching directed topologies," Automatica, vol. 73, pp. 47-55, 2016.

[29] X. Yao, Y. Lian, and J. H. Park, "Disturbance-observer-based event-triggered control for semi-markovian jump nonlinear systems," Applied Mathematics and Computation, vol. 363, Article ID 124597, 2019.

[30] P. Wang, G.-H. Yang, and Y. Pan, "Event-triggered reliable dissipative filtering for nonlinear networked control systems," Neurocomputing, vol. 360, pp. 120-130, 2019.

[31] J. Lin, L. Li, M. Bi, J. Li, M. Hu, and W. Hu, "A study on performance improvement of IMDD-UFMC with modified $k$ means non-uniform quantization," Optics Communications, vol. 476, Article ID 126324, 2020.

[32] A. G. Dimitrov, J. P. Miller, Z. Aldworth, and T. Gedeon, "Non-uniform quantization of neural spike sequences through an information distortion measure," Neurocomputing, vol. 38-40, pp. 175-181, 2001.

[33] T. Kitayabu, H. Ishikawa, M. Hagiwara, and H. Shirai, "Effect of input-signal statistical property in delta-sigma modulator with non-uniform quantization," in Proceedings of the 2012 IEEE Radio and Wireless Symposium, pp. 183-186, Santa Clara, CA, USA, January 2012.
[34] S. Liu, L. Xie, and D. E. Quevedo, "Event-triggered quantized communication-based distributed convex optimization," IEEE Transactions on Control of Network Systems, vol. 5, no. 1, pp. 167-178, 2016.

[35] X.-L. Hu, "An extension of young's inequality and its application," Applied Mathematics and Computation, vol. 219, no. 12, pp. 6393-6399, 2013. 